\title{
NUMERICALSIMULATIONS OF PERIODIC VORTEX SHEDDING FORMED IN THE WAKE OF AN OBJECT PLACED IN V- SHAPED NARROW CHANNEL
}

\author{
Anil Kumar Sharma1', J. Anandan, M.Kumaresan², Lydia Gnanadhas³, \\ B.K. Nashine 4 , P. Chellapandi 5 \\ Indira Gandhi Centre for Atomic Research, Kalpakkam - 603102, Tamilnadu, India
}

\section{ABSTRACT}

\begin{abstract}
Numerical study has been carried out to understand dynamics of flow past a circular disk placed in narrow $V$ shaped channel for different Reynolds number. The Reynolds number based on the disk diameter is fixed for the study. This basic study will help to understand the frequency of vibration due to formation of vortex shedding and its coincident with natural frequency of the object. The velocity and pressure distribution in the flow domain are obtained from the Navier-Stokes solver, computation of forces and moments on the circular disk. The governing equations are solved by a finite volume method. Pressure-velocity coupling between the incompressible Navier-Stokes and continuity equations is established using the Pressure Implicit with Splitting of Operators algorithm. The first order semi- implicit scheme is chosen for the time integration. The numerical data are compared with inhouse experimentally obtained flow field at different time intervals. It is found that the flow fields obtained numerically are well in line with the experimental data. It is established from the observation and calculated values that the shedding frequency increases with the flow velocity around the body. The findings of this study will help to calculate the periodic force which becomes spatially correlated to the mode shape and large amplitudes and leads to fatigue cracks and fretting wear damage to the engineering equipment such heat exchangers etc.
\end{abstract}

\section{INTRODUCTION}

Component failures due to excessive flow induced vibrations continue to affect the performance of nuclear power plants. Such failures are very costly in terms of repairs and lost production. In general the problems caused by excessive vibration are fatigue cracks and fretting wear damage as reported widely in the literature. Tube failure due to fretting wear in nuclear steam generator and vibration related damage in nuclear fuels are particular concern. For parallel plate type fuel assemblies experiments were performed to analyze the flow induced vibration behavior of a four square fixsupported flexible plate exposed to the axial flow within a rigid narrow channel. Large amplitude and narrow band vibration phenomenon observed in the experiment where flow velocity varied with the range of 0 to $5 \mathrm{~m} / \mathrm{s}$ (Lifang Liu et al., 2011). Fluid elastic instability, most important vibration mechanism for tube bundles subjected to cross flow. Most heat exchanger and steam generator are related to fluid elastic instability. The topic of fluid elastic instability of tube bundles reviewed by Pettigrew and
Taylor (1991). Vibrations response due to turbulence induced excitation in Freon liquid-vapor instead of airwater mixtures as excitation forces are lower in Freon because of lower surface tension and lower liquid-vapor density then air-water (Pettigrew et al., 1995). To validate the design and the adequacy of the support system provided in PFBR intermediate heat exchanger for the IHX, flow induced vibration (FIV) experiments were carried out in a water loop system on a $60^{\circ}$ sector model by Prakash et al., (2009). They concluded that IHX support system is adequate with sufficient margin and design is safe against FIV. Dynamic analysis of the tube support system was carried out using ANSYS code. Tube to tube sheet joint was treated as pinned based on model measurement carried out earlier on three tube model (Prakash et al., 1995). Bed friction plays a critical role in controlling the wake-boundary layer interaction, these effects were quantified and observed that its influence is more pronounced and observed in shallow flows compared to deep flow (Balachandar et al.,1999). They observed the wake region of a bluff body is quite distinct 
in deep flows with kàrmàn vortex street formation, while in a corresponding shallow flow, it was found to be stabilized due to frictional influences and vortex shedding is found to be annihilated. The experimental study on velocity distribution in sub channel was carried out by Gang et al., 2004. The FIV phenomena of fuel plates were examined in a 1:1 model for the wide range of velocity. The velocity distribution within sub-channel, vibration frequency spectrum and displacement of the plate were tested. The Physical Component Boundary Fitted Coordinate method and Finite Difference Method scheme were used in a specially developed code to simulate the FIV characteristic for the simple supported beam model by Juanhua and Daogang (2009), in which vortex shedding characteristics around an elongated rigid plate and vibration displacement of an elongated flexible plate were computed in different cases of flow velocity. However, plate deformation to the flow field was not considered. Narasimhamurthy and Helge (2009), predicted the turbulent wake behind a normal flat plate based on the Navier-Stokes equations and 2nd - order central-differencing scheme for incompressible fluid in case of Reynolds number of 750 . They reported that the coherent vortices were alternately shed from the sides of the plate with a frequency corresponding to a Strouhal number 0.168 and the wake was distinctly turbulent downstream of the plate whereas the mean recirculation bubble extended $1.96 \mathrm{D}$ downstream.

The experimental study by Pettigrew et al. (1978) and also by Gorman (1978) clearly emphasize that the main flow induced vibration (FIV) mechanism in cross flow are periodic vortex shedding, random excitation due to turbulence and the fluid elastic instability. Other than the inlet and outlet, flow is parallel to the tube along the remaining part of the IHX with a weak excitation due to turbulence. In French Super-Phenix nuclear reactor during IHX Flow induced vibration testing, it was observed that instability due to an excessive cross flow velocity in the second span caused by wrong location of support (Technical report 1989). In one of the Japanese nuclear reactor due to improper insertion of anti-vibration belt by fabrication engineer lead to failure of SG tube by FIV and leak of primary radioactive coolant.
From the above literature it is clear that considerable progress has been made in the area of flow induced vibration. However, the literature on large amplitude and narrow band vibration phenomena of an objects placed in triangular rigid narrow channels is scare. The present study is concerned about the periodic vortex shedding formed in the wake of an objects placed in triangular rigid narrow channels. This basic experimental study will help to understand the frequency of vibration due to formation of vortex shedding and its coincident with natural frequency of the object. This in turn will help to calculate the periodic force which becomes spatially correlated to the mode shape and large amplitudes, which could lead to fatigue cracks and fretting wear, damage to the engineering equipment such heat exchangers etc.

\section{EXPERIMENTAL SET UP:}

Experiments were carried out in a water loop system as shown in the Fig. 1.The disk of diameter $25 \mathrm{~mm}$ and thickness $12 \mathrm{~mm}$ about which water flows is placed in the triangular cross section. The water is made to flow inside a V-shaped channel of thickness $16 \mathrm{~mm}$ as shown in Fig. 2. For this purpose a motor is introduced in the loop to maintain a constant flow rate in the water loop system and circulate it. The flow rate through the loop is also measured to know the flow velocity across the section.

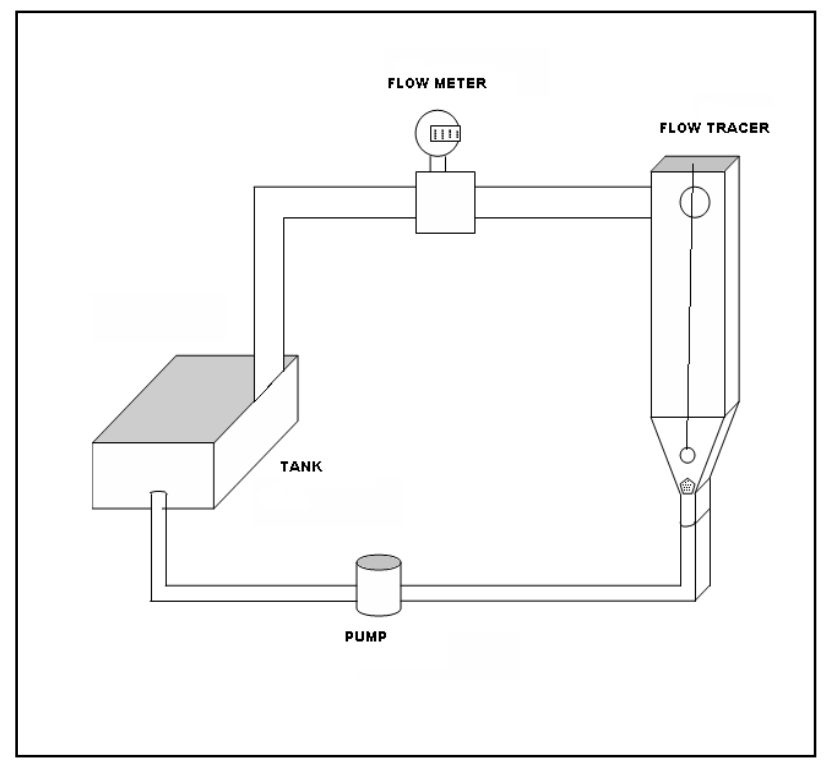

Fig.1. Schematic of Experimental Test set up 
In the experiment, the vortex shedding due to the circular disk placed in channel is detected by a high speed recording camera at different positions of the disk inside V-section with time

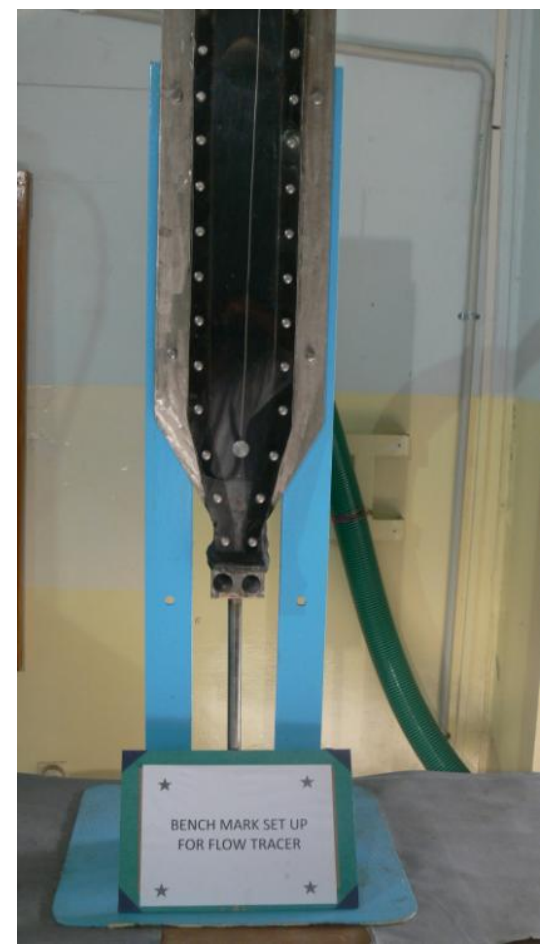

All dimensions in

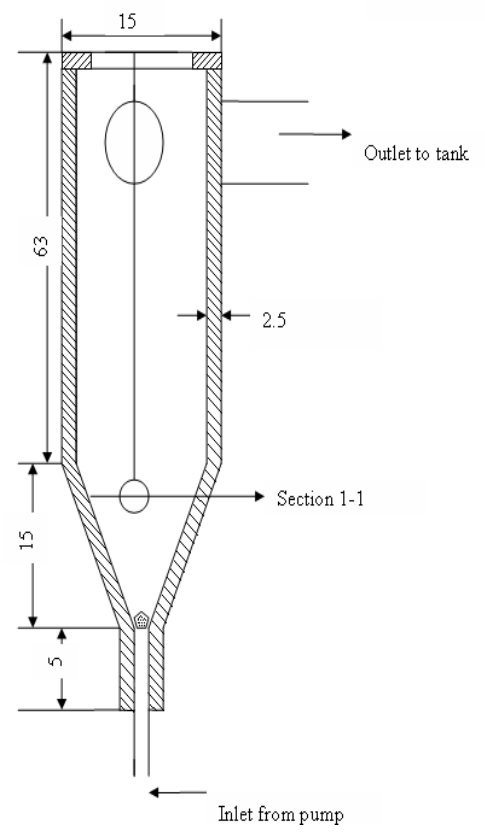

Fig. 2. Front view of model and image of the set up

\section{GOVERNING EQUATIONS AND SOLUTION METHODOLOGY:}

\section{A. Governing Equations}

The fluid flow past a circular disk in two dimensions is governed by the time-dependent incompressible form of Navier-Stokes and continuity equations with Newtonian approximation. The governing equations in Cartesian coordinate system can be written as (Reddy and Gartling, 2000),

$$
\begin{aligned}
& \frac{\partial u}{\partial x}+\frac{\partial v}{\partial y}=0 \\
& \left(\frac{\partial u}{\partial t}+u \frac{\partial u}{\partial x}+v \frac{\partial u}{\partial y}\right)=-\frac{1}{\rho} \frac{\partial p}{\partial x}+v\left(\frac{\partial^{2} u}{\partial x^{2}}+\frac{\partial^{2} u}{\partial y^{2}}\right) \\
& \left(\frac{\partial v}{\partial t}+u \frac{\partial v}{\partial x}+v \frac{\partial v}{\partial y}\right)=-\frac{1}{\rho} \frac{\partial p}{\partial y}+v\left(\frac{\partial^{2} v}{\partial x^{2}}+\frac{\partial^{2} v}{\partial y^{2}}\right)
\end{aligned}
$$

Here, $u$ and $v$ refer to velocities in the two component directions, and $p$ refers to pressure. Fluid properties are density, $\rho$ and kinematic viscosity $u$.

\section{B. Initial and Boundary Conditions}

The flow domain of interest is depicted in Fig.2. The boundary conditions for two-dimensional simulations are:

a) Inlet located at a distance of 4D on the upstream of the disk, is imposed with a uniform velocity $U_{\infty}$

b) Outlet located at a distance of 14D downstream of the cylinder with zero traction and a constant pressure

c) No-slip boundary condition on the disk wall, and

d) Free-slip condition on the side confining channel walls

\section{Numerical Approach}

The governing equations (1) - (3) are solved by a finite volume method using a general purpose computational fluid dynamics (CFD) tool. Pressurevelocity coupling between the incompressible Navier- 
Stokes and continuity equations is established using the Pressure Implicit with Splitting of Operators (PISO) algorithm (Versteeg and Malalasekara, 1995. The first order semi-implicit scheme is chosen for the time integration. In the PISO solver, the pressure term is treated implicitly at $(n+1)^{\text {th }}$ time level, while all other terms are treated explicitly at $(\mathrm{n})^{\text {th }}$ time level as follows:

$$
u^{n+1}=u^{n}-\Delta t\left[\frac{\partial p}{\partial x_{i}}\right]^{n+1}
$$

Since it is not possible to compute both velocity and pressure simultaneously at $(n+1)^{\text {th }}$ time level, the above equations are split into one that computes an intermediate pressure $p^{*}$, and an intermediate velocity $u_{i}^{*}$ as follows:

$u_{i}^{*}=u_{i}^{n}-\Delta t\left\{u_{j} \frac{\partial u_{i}}{\partial x_{j}}\right\}^{n}-\Delta t\left(\frac{\partial p^{*}}{\partial x_{i}}\right)$

However, we need to project these intermediate velocities (which do not satisfy mass conservation) into a divergence free vector space. Subtract Eq. (5) from Eq. (4) to get

$$
u_{i}^{n+1}-u_{i}^{*}=-\Delta t\left\{\frac{\partial^{2} p^{n+1}}{\partial x_{i} \partial x_{i}}-\frac{\partial p^{*}}{\partial x_{i}}\right\}
$$

Apply divergence on both sides of Eq. (6), and enforce mass conservation $\partial u_{i}^{n+1} / \partial x_{i}=0$ to obtain the following Poission equation

$$
\nabla^{2} p^{n+1}-p^{*}=-\frac{1}{\Delta t} \frac{\partial u_{i}^{*}}{\partial x_{i}}
$$

It should be noted that, the intermediate value of pressure $\left(p^{*}\right)$ can be evolved through an initial guess pressure. Further details related to the flow solver and spatial and discretization scheme are available in detail in references, Patankar, 1980. The discrete temporal step $\Delta t$ is determined by the CFL (Courant-Friedrichs-Lewy) criterion (Fletcher, 1987). Setting convergence criterion becomes rather difficult, due to the unsteady and periodic nature of the wake region. However, an absolute error $(\varepsilon)$ in the discretized momentum and continuity equations is set using a tolerance criterion as, $\varepsilon^{<10^{-4}}$.

\section{RESULTS AND DISCUSSIONS:}

\section{A. Grid sensitivity check}

In any CFD simulation, establishing the independent nature of the solution on the grid size used in the calculations is an essential first step. Towards this goal, an attempt is made to determine optimum mesh for two dimensional simulations. It is observed that the number of radial grid divisions on the periphery of the cylinder is a useful parameter to determine the mesh for accurate prediction of drag and lift forces. Grid convergence tests are carried out first for the case of $\operatorname{Re}=1400$, with three different mesh sizes. Under two dimensional settings, the flow domain of interest is tessellated with a nonhomogeneous grid as shown in the Fig. 3(a). A close-up view of the mesh, shown in Fig. 3(b), reflects the fine mesh closer to the no-slip surfaces. Different mesh sizes employed for the grid sensitivity study are shown in Table 1. Here, the force coefficients, Strouhal number, CPU time requirements associated with grid sizes are presented. It can be noticed that the difference between the results obtained with mesh M3 and M2 is very small, thus justifying the use of the M3 mesh for the present study. Spatio-temporal dynamics of the wake behind the circular disk at $\operatorname{Re}=1400$ for mesh of size $M 3$ are presented in Fig. 4(a) and 4(b) respectively. The alternate nature of vortex shedding is well captured. 


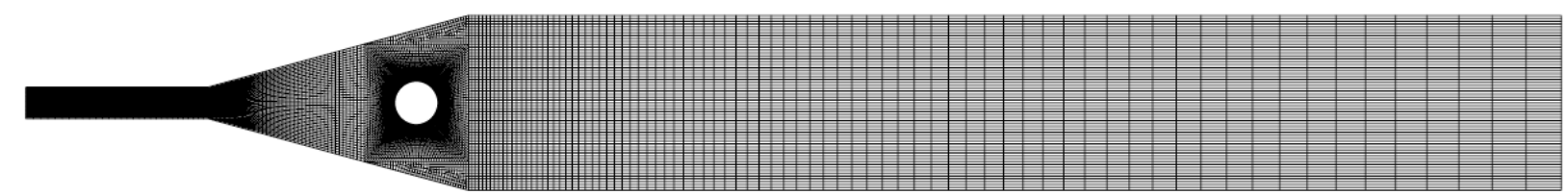

(a) Global grid pattern

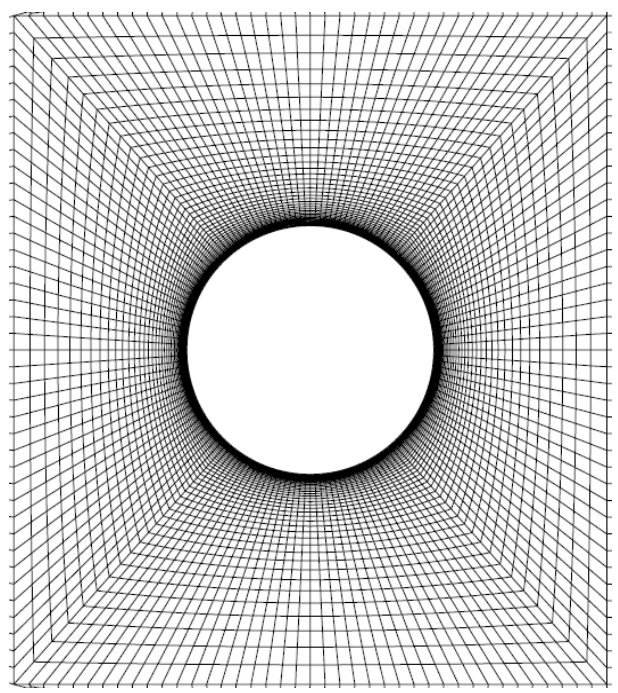

(b) Close-up view around the disk

Fig.3 (a-b): Global grid pattern and close-up view around the disk

Table - 1: Grid sensitivity study for $\operatorname{Re}=200$

\begin{tabular}{|c|c|c|c|c|}
\hline Mesh & $\begin{array}{c}\text { No. of control } \\
\text { volumes }\end{array}$ & $C_{D}$ & $C_{L}$ & $\begin{array}{c}\text { CPU time per iteration } \\
\text { (s) }\end{array}$ \\
\hline M1 & 9478 & $0.11 \pm 0.01$ & \pm 0.1 & 4 \\
\hline M2 & 14402 & $0.1 \pm 0.009$ & \pm 0.09 & 5 \\
\hline M3 & 19608 & $0.1 \pm 0.0006$ & \pm 0.08 & 7 \\
\hline
\end{tabular}
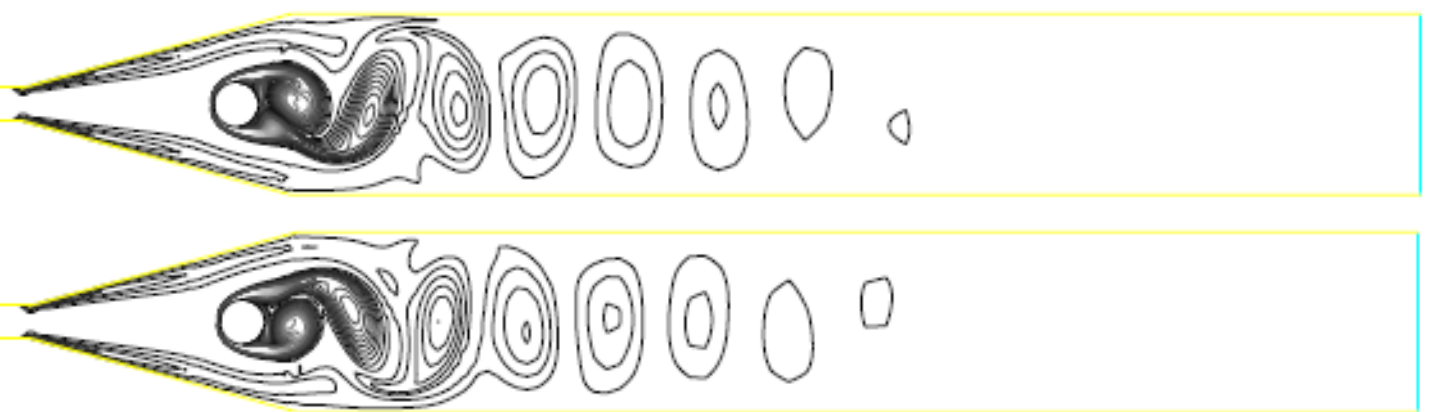

(a) 


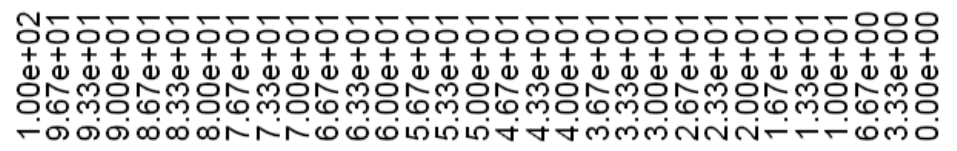

(b)

Fig. 4: Contours depicting alternate shedding at different times: (a) $22.7 \mathrm{~S} \&$ (b) $33.7 \mathrm{~S}$

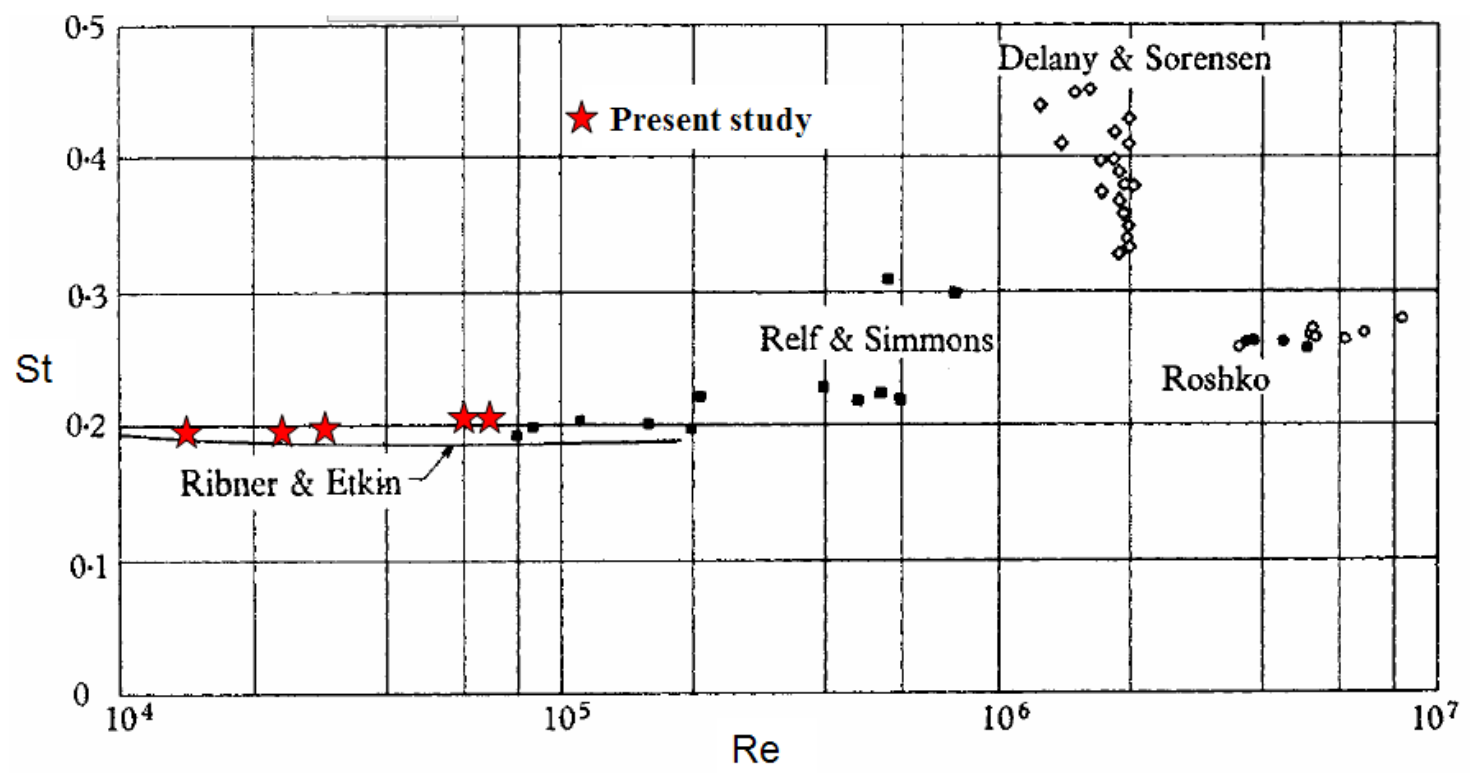

Fig. 5. Comparison of Strouhal number Vs Reynold number with available results

\section{B. Comparison of the Obtained Results}

The Strouhal number of the vortex shedding frequency is plotted against Reynold number in Fig. 5, together with previous measurements available in the literature. It can be seen that the results are in very good agreement with the published data. This gives the confident to carry out the advanced research in the field of FIV for complex structural geometries with different flow situations.

\section{Vortex Shedding}

To understand the dynamics of flow past a circular disk placed in narrow $\mathrm{V}$ shaped channel the disk of 25 $\mathrm{mm}$ diameter is considered for different Reynolds number. The Reynolds number based on the disk diameter is fixed for the study. The velocity and pressure distribution in the flow domain are obtained from the
Navier-Stokes solver, computation of forces and moments on the circular disk. Figure 6 depicts the numerically and experimentally obtained flow field at different time intervals. It is clear from these figures that the flow fields obtained numerically are well in line with the experimental data. Periodic wake shedding found to occur immediately downstream of the disk when subjected to flow through channel. It is evidence from figure 7 that the periodic wake shedding is generated, which in-turn leads to periodic fluid forces. This periodic fluid force leads to excessive vibration amplitudes.

It is also clear from these figures that the flow around the disk is slowed down while in contact with its surface and forming the boundary layer and this boundary layer found to be separated from the disk due to curvature of the disk. Vortices are then formed changing the pressure distribution along the surface. The vortices are not formed 
symmetrically around the disk and hence different lift forces develop on each side of the body, thus leading to motion of transverse to the flow. This motion is found to change the nature of vortex formation in such a way as to lead to limited amplitude. This leads to excessive vibration amplitudes as explained earlier and finally leads to damage the structures. The lift and drag forces per unit axial length are obtained by integrating the normal and tangential stresses, which are due to pressure and velocity gradients as given in Fig 8 . It is found that the $C_{D}$ and $C_{L}$ values are in the range of $0.1 \pm 0.009$ and \pm 0.09 for the specific $\operatorname{Re}=1400$.

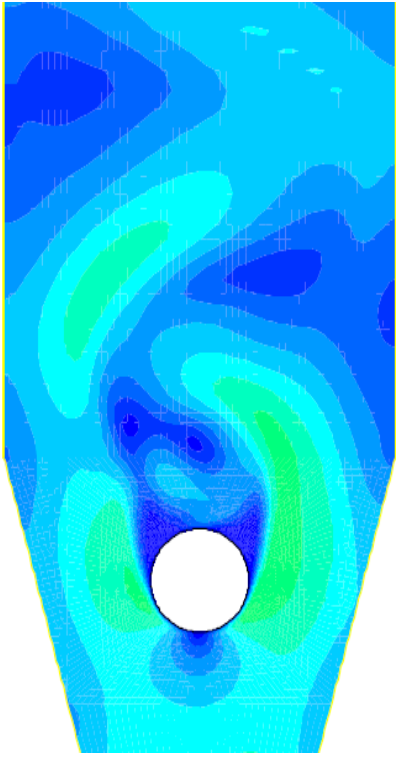

(Numerical)

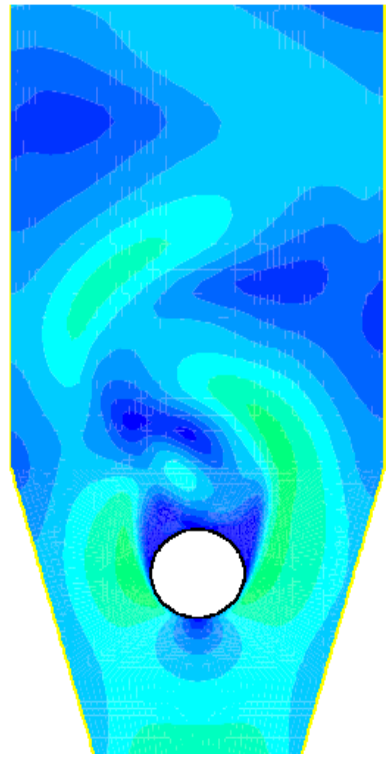

(Numerical)

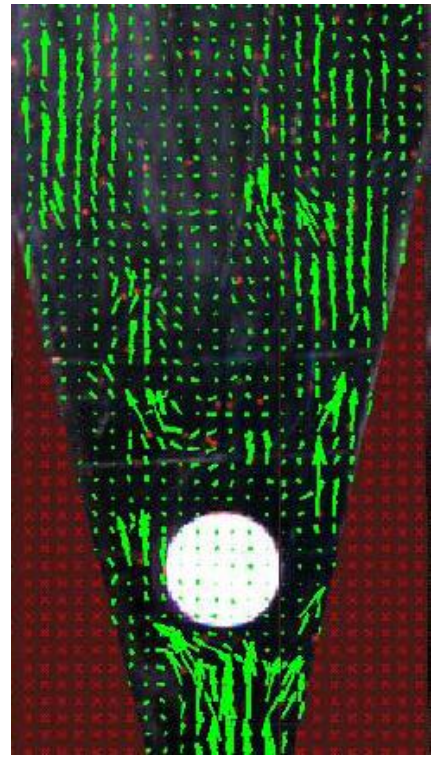

(Experimental)

(a) at $100 \mathrm{~ms}$

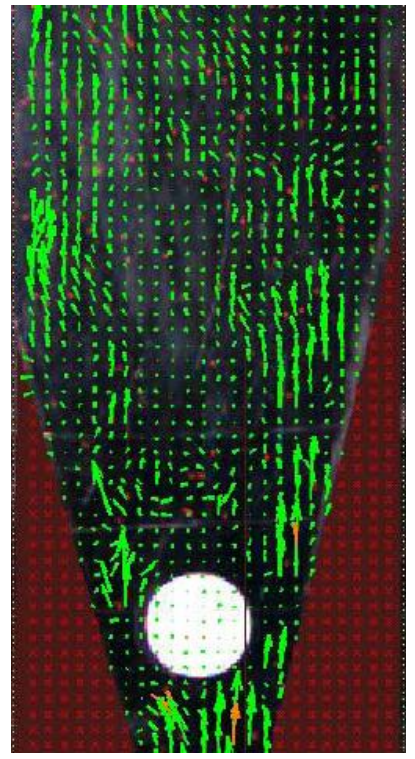

(Experimental)

(b) $200 \mathrm{~ms}$ and 


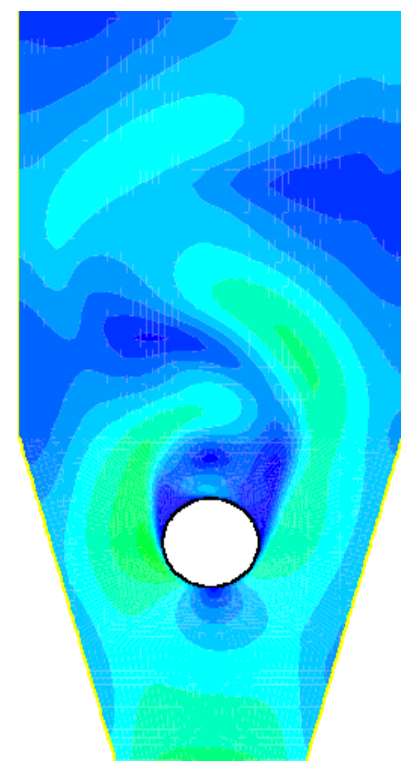

(Numerical)

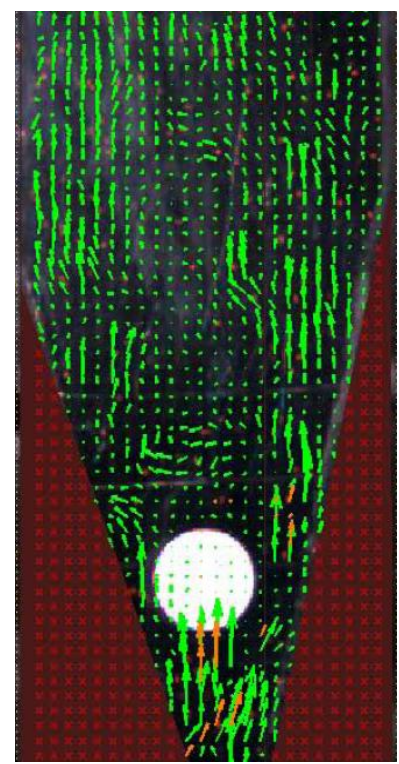

(Experimental)

(c) $300 \mathrm{~ms}$ respectively

Fig. 6 Velocity contours (Numerical) and experimental obtained flow field at different time intervals (a) at $100 \mathrm{~ms}$ (b) $200 \mathrm{~ms}$ and (c) $300 \mathrm{~ms}$ respectively

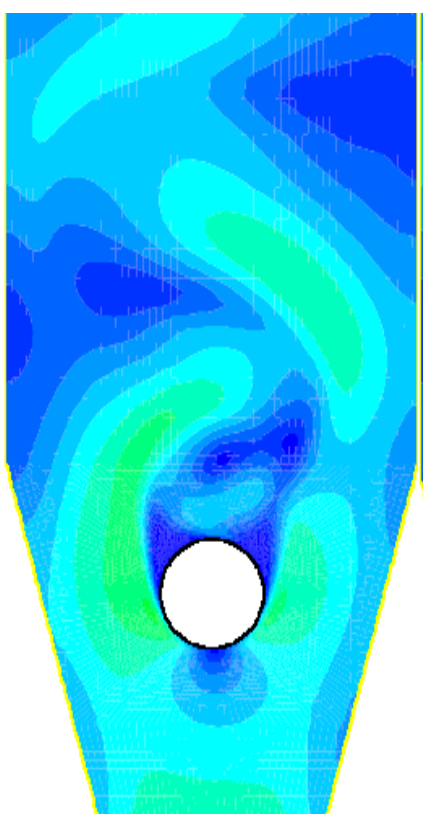

(a)

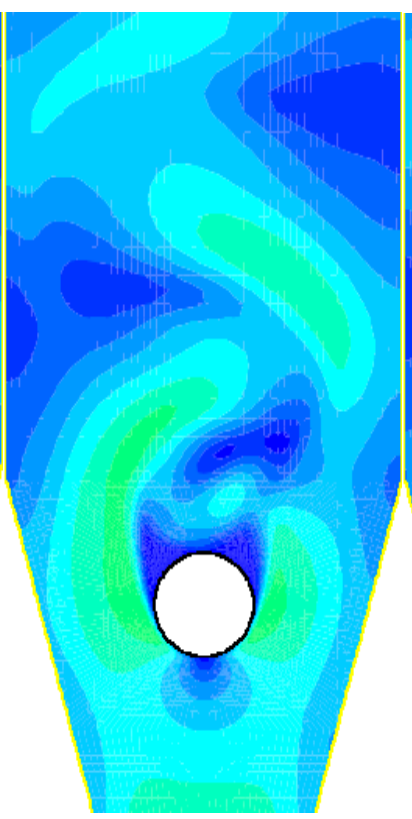

(b)

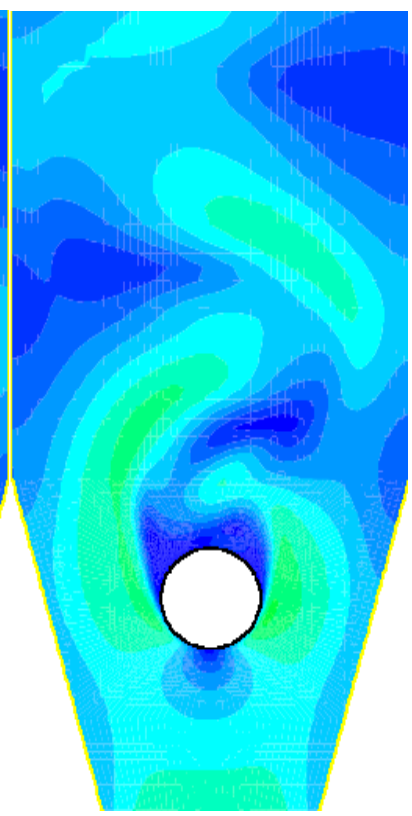

(c)

Fig.7 (a-c) Velocity contours at different time intervals (a) at $1000 \mathrm{~ms}$ (b) $2000 \mathrm{~ms}$ and (c) $3000 \mathrm{~ms}$ respectively 


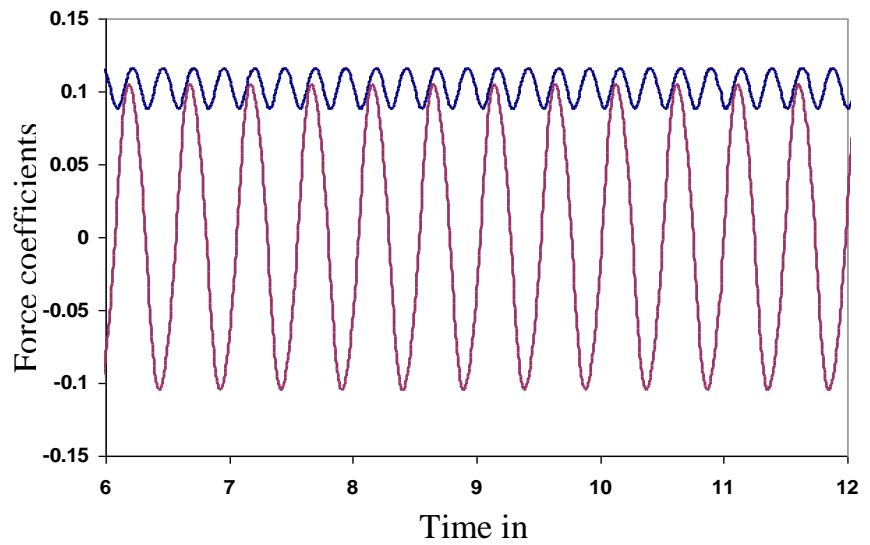

Fig. 8: Lift and Drag coefficients for $\operatorname{Re}=1400$

\section{CONCLUSION:}

The present study is concerned about the periodic vortex shedding formed in the wake of an object placed in triangular rigid narrow channel. The series of experiments and numerical analysis are carried out to understand the frequency of vibration due to formation of vortex shedding and its coincident with natural frequency of the object. The governing equations are solved by a finite volume method. Pressure-velocity coupling between the incompressible Navier-Stokes and continuity equations is established using the Pressure Implicit with Splitting of Operators algorithm. The first order semi- implicit scheme is chosen for the time integration. The vortex shedding frequency for different flow velocity is been calculated and verified. From the observation and calculated values, the shedding frequency increases with the flow velocity around the body. The findings of this study will help to calculate the periodic force which becomes spatially correlated to the mode shape and large amplitudes and leads to fatigue cracks and fretting wear damage to the engineering equipments.

\section{REFERENCE}

[1] Roshko, 1954. On the drag and shedding frequency of bluff cylinders. Nut. Adv. Comm. Aero., Wash., Tech. Note 3169.

[2] A. J. Fletcher, Computational Techniques for Fluid Dynamics-2: Specific Techniques for different Flow Categories, Springer Publications, 1987.

[3] E. F. Relf, L. F. G Simmons, 1924. The frequency of eddies generated by the motion of circular cylinders through a fluid. Aero. Res. Counc., Lond., Rep. and Mem. no. 917.

[4] H. K. Versteeg and W. Malalasekara, An Introduction to Computational Fluid Dynamics, $2^{\text {nd }}$ edition, Pearson Education, 1995.

[5] H. S. Ribner, B.Etkin, 1958. Noise research in Canada. Proc. 1st Int. Congr. Aero Sci., Madrid (publ. by Pergamon Press, London, 1959).

[6] J. N. Reddy and D. K. Gartling, The Finite Element Method in Heat Transfer and Fluid Dynamics, $2^{\text {nd }}$ edition, CRC Press, 2000.

[7] K. Satpathy. K. Velusamy, B.S.V.Patnaik P. Chellapandi, 2011. Numerical investigation of vortex shedding past a finite circular cylinder mounted on a flat plate. Numerical heat transfer, Part A, 59 : 882-909.

[8] Lifang Liu, Daogang Lu, Yang Li, Fengli Niu, 2011. Largeamplitude and narrow-band vibration phenomenon of failure of a foursquare fix-supported flexible plate in a rigid narrow channel. Nuclear Engineering and Design 241, 2874-2880.

[9] M. Gang, B. Hanliang, J. Shengyao, 2004. Flow induced vibration in parallel-plate fuel assemblies. Tsinghua University (sci \&Tech) Vol.44 (3), 350-353.

[10] M. J. Pettigrew C.E. Taylor, J.H. Jong, I.G. Currie, 1995.Vibrations of a triangular tube bundle in two-phase Freon cross flow.ASME J. press. Vessel technology.117 (4), 321-329.

[11] M.J. Pettigrew, C. E. Taylor, N.J. fisher, M. Yetisir, B.A.W. Smith,1998. Flow-induced vibration: recent findings and open questions. Nuclear engineering and Design 185, 249276.

[12] N. K. Delany, N. E. Sorensen, 1953. Low-speed drag of cylinders of various shapes. Nat. Adv. Comm. Aero., Wash., Tech. Note 3038.

[13] R. Balachandar, M. F. Tachie, and V. H. Chu, 1999. Concentration profile in shallow wakes. Trans ASME. J. Fluid Engg., (121), 34-43.

[14] S. V. Patankar, Numerical Heat Transfer and Fluid Flow, Hemisphere, New York, 1980.

[15] V. Prakash, M. Thirumalai, R. prabhakar, G. Viadyanathan, 2009. Assessment of flow induced vibration in sodiumsodium heat exchanger. Nuclear engineering and Design 239, 169-179.

[16] V. Prakash.,et al., 1995. Modal Measurement of on PFBR components. National workshop on shock and Vibration , Hyderabad, India.

[17] V.D. Narasimhamurthy, I.A. Helge, 2009. Numerical Simulation of the turbulent of the turbulent wake behind a normal flat plate. Int.J.Heat fluid flow 30, 1037-1043. 